\title{
High Sensitivity CRP and Short-Term Cardiovascular Risk among Patients with Acute Myocardial Infarction: A Two-center Study
}

\author{
Richard Henry Perlas Tiongco II, ${ }^{1}$ Camilo G. Te Jr., ${ }^{1}$ Felix Eduardo R. Punzalan, ${ }^{1}$ \\ Celia Catherine C. $\mathrm{Uy}^{1}$ and Von Meldrick A. Gonda ${ }^{2}$ \\ ${ }^{1}$ Section of Cardiology, Department of Medicine, College of Medicine and Philippine General Hospital, University of the Philippines Manila \\ ${ }^{2}$ Makati Medical Center, Makati City
}

\begin{abstract}
Objective. To determine high sensitivity C-reactive protein (hsCRP) levels of patients admitted for myocardial infarction (MI) and determine its association with existing traditional cardiovascular (CV) risk factors and future cardiovascular events.

Methods. This was a prospective cohort study involving two centers, the Makati Medical Center and the University of the Philippines-Philippine General Hospital (UP-PGH). A total of 49 acute MI patients who fulfilled the inclusion and exclusion criteria were enrolled in the study. Levels of hs-CRP were taken within 6 hours of admission. Demographic data, in-patient outcomes and 30-day clinical outcomes were observed. A univariate analysis with Fisher's Exact Test was done to determine if there was an association between hs-CRP levels and traditional CV risk factors, and clinical outcomes.
\end{abstract}

Results. There was a significantly higher difference in the primary composite endpoint of all-cause mortality, MI, unstable angina and revascularization among patients with hs-CRP> $10 \mathrm{mg} / \mathrm{dL}(\mathrm{Cl}$ : $95 \%, p=0.045)$.

Conclusion. The study shows that elevated levels of hs-CRP can be utilized for cardiovascular risk stratification and prognostication among patients with acute myocardial infarction.

Key Words: hs-CRP, myocardial infarction, coronary artery disease

\section{Introduction}

Time has afforded medicine the opportunity to develop improvements in the quality of care of patients who experience acute coronary events, particularly acute myocardial infarction (AMI). With the advent of thrombolysis, and later revascularization, such medical advances have reached new grounds in defining survival benefit and improvement in the quality of life of our

Corresponding author: Richard Henry Perlas Tiongco II, MD

Department of Pharmacology and Toxicology

College of Medicine

University of the Philippines Manila

Telephone: +6325251841

Email: rickytmd@yahoo.com patients. With further advances in medicine, recent paradigms have begun to concentrate on the "vulnerable plaque" and pathophysiological processes of inflammation, in order to further optimize patient care.

High sensitivity C-reactive protein (hs-CRP) is one chemical marker that has earned much attention in profiling patients with coronary disease. As some literature has spawned its use in prognosticating chronic stable angina, recent studies cite its use in profiling the future clinical courses of patients with acute myocardial infarction. In the TACTICS-TIMI 18 studies concerning NSTEMI/Unstable Angina, women were more likely to have elevated hs-CRP; men on the other hand were more likely to have elevated creatinine kinase-MB and troponins. Such gender-related differences need further studies for verification in the setting of acute coronary syndromes. Across the acute coronary syndromes, elevated CRP levels are associated with an increased risk of recurrent events, independent of the presence or absence of myocyte necrosis.

Recent studies have looked at hs-CRP not just as a marker of inflammation but also as a mediator in the inflammatory process, and thus linking hs-CRP directly to atherothrombotic effects. CRP has been correlated with: the promotion of LDL uptake by macrophages, the induction of tissue factor by monocytes, the direct induction of another inflammatory mediator monocyte chemo-attractant protein1 (MCP-1), and vascular-cell adhesion molecules (VCAM) in serum by the endothelium. A large prospective, nested case-control study in apparently healthy postmenopausal women over three years revealed that among 12 markers of inflammation studied, hs-CRP was the strongest univariate predictor of the risk of cardiovascular events (death from coronary heart disease, nonfatal myocardial infarction or stroke, or a coronary-revascularization).

The JUPITER study was a large, multi-center, randomized, placebo-controlled primary prevention trial which used an hs-CRP level of $>2.0 \mathrm{mg} / \mathrm{l}$ as a marker of higher cardiovascular risk. In the trial, reduction of hs-CRP using rosuvastatin showed a significant risk reduction in cardiovascular outcomes among the population studied. 
This further strengthened hs-CRP as a novel cardiac biomarker for risk stratification and as a target for treatment.

In pre-interventional intravascular ultrasound studies (IVUS) of patients with acute myocardial infarction, there was significantly more plaque rupture in patients with elevated hs-CRP, suggesting that elevated hs-CRP levels may reflect the inflammatory activity of a ruptured plaque and/or the plausible intensification of focal inflammatory processes that destabilize vulnerable plaques. High sensitivity C-reactive protein has also been correlated with poor short and long term prognosis in the period after an acute coronary event.

The profiling of patients admitted for acute myocardial infarction in the local setting will provide physicians with essential information to regional and racial values of $\mathrm{C}$ reactive protein. The information thus gained may then aid local physicians in developing treatment strategies or protocol in addressing the inflammatory process in the setting of acute myocardial infarction. One example is the recent evidence that statins and peroxisome-proliferatoractivated receptor-alpha promoters (fibrates) can inhibit the pro-inflammatory effect of hs-CRP. With all these in mind, the data will provide essential information perhaps not only for prognostication but also for future studies and the improvement of treatment strategies in the setting of acute myocardial infarctions.

\section{Objectives}

- Primary objective: To determine high-sensitivity CRP levels in patients admitted for acute myocardial infarction at the Makati Medical Center and the University of the Philippines-Philippine General Hospital.

- Secondary objectives:

- To determine the association between CAD risk factors and hs-CRP levels taken during admission.

- To determine the association of hs-CRP levels in patients admitted for acute myocardial infarction and future cardiovascular events such as acute myocardial infarction, congestive heart failure, revascularization and all-cause mortality, over a 30day period post myocardial infarction.

\section{Methods}

This is a prospective study of AMI patients admitted at the University of the Philippines-Philippine General Hospital and the Makati Medical Center for acute myocardial infarction from February 1, 2005 to August 31, 2005.

The study population was interviewed by a physician who completed a standard data collection form. All patients gave an informed consent and the study was approved by the hospitals' respective ethics committees.
Serum hs-CRP, fasting blood sugar and lipid profile were extracted from these subjects within 6 hours of diagnosis. Serum hs-CRP levels were determined by using the Immulite 1000 hs-CRP machine manufactured by Diagnostic Products Corporation. Processing was done at Best Diagnostics, Quezon City. The demographic data, inpatient mortality and morbidities were noted, and 30-day clinical outcomes were obtained by phone interview with the patient or family members. The variables of interest are:

1. Level of hs-CRP

2. Incidence of complications (including recurrence of an acute coronary syndrome or congestive heart failure) and mortality for patients admitted for acute myocardial infarction.

3. Cardiovascular morbidity within 30 days post acute myocardial infarction:

a. Recurrence of an acute coronary syndrome (myocardial infarction or Unstable angina)

b. Progression into congestive heart failure (symptoms fulfilling the Framingham Criteria; with Left Ventricular Ejection Fraction $<40 \%$ )

4. Cardiovascular causes of mortality and morbidity (recurrent acute coronary syndrome and/or congestive heart failure).

5. The levels of hs-CRP and the need for coronary revascularization.

\section{Inclusion Criteria}

Myocardial infarction: (Based on 2000 WHO criteria), at least two criteria must be present:

(1) typical (ischemic) symptoms of prolonged (>20 minutes) chest pain

(2) Serial ECG changes

(a) progression from no $Q$ wave to a definite $Q$ wave

(b) a lesser $\mathrm{Q}$ wave progression combined with progressive ST-segment depression, developing ST-segment elevation, or progressive T-wave inversion

(c) persistent ST-segment elevation with progressive $\mathrm{T}$-wave inversion in sequential daily ECGs

(3) cardiac enzyme levels (Creatine kinase-MB) twice the limit of normal (with reference to the local laboratory upper limit of normal values)

\section{Exclusion Criteria}

1. Prior myocardial infarction ( $<1$ month from present admission)

2. Inflammatory diseases

3. Trauma
a. surgery
b. burns 
c. fractures

4. Infections
a. bacterial
b. severe fungal
c. viral

5. Allergic complications of infection
a. rheumatic fever
b. erythema nodosum

6. Necrotic conditions

a. tumor embolization

b. acute pancreatitis

7. Malignancy
a. lymphoma
b. carcinoma
c. sarcoma

8. Other conditions:
a. systemic lupus erythematosus
b. dermatomyositis
c. ulcerative colitis
d. leukemia
e. graft vs. host disease

9. Patients whose time from onset of symptoms to admission to the emergency room unit is $>6$ hours

10. Creatinine $>176 \mathrm{umol} / 1$

\section{Definition of Variables}

I. Congestive Heart Failure: Presence of two major or one major and two minor criteria; minor criteria acceptable only if they could not be attributed to another medical condition (Framingham criteria, c.1993); LVEF 40\%

\section{Major Criteria}

a. Paroxysmal nocturnal dyspnea

b. Neck vein distension

c. Rales

d. Radiographic cardiomegaly

e. Acute pulmonary edema

f. S3 gallop

g. Central venous pressure $>16 \mathrm{~cm} \mathrm{H} 2 \mathrm{O}$

h. Circulation time $>/=25$ secs

i. Hepatojugular reflux

j. Pulmonary edema, visceral congestion, (or cardiomegaly at autopsy)

k. Weight loss $>/=4.5 \mathrm{~kg}$ in 5 days in response to treatment of congestive heart failure

\section{Minor Criteria}

a. Bilateral ankle edema

b. Nocturnal cough

c. Dyspnea on ordinary exertion

d. Hepatomegaly

e. Pleural effusion f. Decrease in vital capacity by $1 / 3$ from the maximal value recorded

g. Tachycardia (rate $>/=120$ beats $/ \mathrm{min}$.)

II. Hypertension (Blood pressure noted to be as follows: Systolic BP $>/=140$; Diastolic $\mathrm{BP}>/=$ 90; based on JNC VI)

III. Dyslipidemia (HDL < 40 mg/dl; LDL > 100 mg/dl; levels defined in NCEP-ATP III)

IV. Presence/absence of Diabetes Mellitus (FBS > 126 taken twice; symptoms of diabetes/hyperglycemia with RBS > 200; based on 1997 ADA Guidelines)

V. Presence/absence of Impaired Fasting Glycemia $($ FBS $=>110$ but $<126 \mathrm{mg} / \mathrm{dl} ; 1997$ ADA Guidelines)

VI. Smoking history (Current or previous smoking history)

VII. Obesity (BMI $>/=25 \mathrm{~kg} / \mathrm{m} 2$ per Asia Pacific Conference standards)

VIII. History of premature coronary heart disease in the Family (men ages <55; women ages <65) (Braunwald's Heart Disease, $8^{\text {the }}$ )

IX. History of Metamphetamine use

\section{Statistical Analyses}

Univariate analysis by Fisher's exact test was used for categorical variables. For all analyses, significance was defined as $\mathrm{p}$ value $<0.05$, two-tailed test.

\section{Results}

Forty nine patients were enrolled in our study and baseline characteristics were tabulated in Table1.

Table 1. Baseline clinical characteristics

\begin{tabular}{ll}
\hline 49 MI patients enrolled & \\
\hline Male & $55.1 \%(27)$ \\
Hypertensive & $56.52 \%(26)$ \\
Dyslipidemia & $65.12 \%(28)$ \\
Diabetic & $39.13 \%(18)$ \\
Smoking & $65.96 \%(31)$ \\
Obesity & $19.57 \%(9)$ \\
Premature CHD Hx & $23.91 \%(11)$ \\
MAP Use & $4.55 \%(2)$ \\
\hline
\end{tabular}

In our study, smoking was the only traditional cardiovascular risk factor that was significantly associated with a higher level of hs-CRP using the cutoff value of 10 mg/L. (Table 2)

The American Heart Association (AHA) and the Centers for Disease Control and Prevention (CDC) stratify CAD risk based on hs-CRP levels: low risk $(<1.0 \mathrm{mg} / \mathrm{L})$, moderate risk (1.0-3.0 mg/L), and high risk $(>3.0 \mathrm{mg} / \mathrm{L})$. Using the CDC recommendations for hs-CRP interpretation, smoking and familial history of premature CHD showed significant positive association with higher hs-CRP levels. (Table 3) 
Table 2. Association of high hs-CRP levels and traditional risk factors

\begin{tabular}{lrrl}
\hline CRP Level & $<\mathbf{1 0 ~} \mathbf{~ g} / \mathbf{L}$ & $>\mathbf{1 0} \mathbf{~} \mathbf{g} / \mathbf{L}$ & \\
\hline Male Sex & $57.58 \%$ & $50.00 \%$ & $\mathrm{p}=0.761$ \\
Hypertension & $56.25 \%$ & $57.14 \%$ & $\mathrm{p}=1.000$ \\
Dyslipidemia & $65.52 \%$ & $64.29 \%$ & $\mathrm{p}=1.000$ \\
Diabetes & $37.50 \%$ & $42.86 \%$ & $\mathrm{p}=0.753$ \\
Smoking History & $52.17 \%$ & $79.17 \%$ & $\mathrm{p}=0.069$ \\
Obesity & $25.00 \%$ & $7.14 \%$ & $\mathrm{p}=0.240$ \\
Premature CHD Hx & $28.13 \%$ & $14.29 \%$ & $\mathrm{p}=0.460$ \\
MAP Use & $3.33 \%$ & $7.14 \%$ & $\mathrm{p}=0.540$ \\
\hline
\end{tabular}

(Fisher's exact T-test, 95\% C.I) $\alpha .05$

Table 3. Association of hs-CRP levels (CDC cutoffs) and traditional CV risk factors

\begin{tabular}{|c|c|c|c|c|}
\hline CRP Level & $\begin{array}{l}<1 \mathrm{mg} / \mathrm{L} \\
(\mathrm{n}=17)\end{array}$ & $\begin{array}{l}1-3 \mathrm{mg} / \mathrm{L} \\
(\mathrm{n}=5)\end{array}$ & $\begin{array}{l}>3 \mathrm{mg} / \mathrm{L} \\
(\mathrm{n}=27)\end{array}$ & \\
\hline Male & $41.18 \%(7)$ & $75.00 \%(3)$ & $62.96 \%(17)$ & $p=0.301$ \\
\hline Hypertension & $58.82 \%(10)$ & $25.00 \%(1)$ & $60.00 \%(15)$ & $\mathrm{p}=0.411$ \\
\hline Dyslipidemia & $58.82 \%(10)$ & $50 \%(2)$ & $69.57 \%(16)$ & $\mathrm{p}=0.910$ \\
\hline Diabetes & $35.29 \%(6)$ & $50 \%(2)$ & $40.00 \%(10)$ & $\mathrm{p}=0.411$ \\
\hline Smoking & $21.43 \%(3)$ & $50 \%(2)$ & $76 \%(19)$ & $\mathrm{p}=0.000$ \\
\hline Obesity & $35.29 \%(6)$ & $0.00 \%$ & $12.00 \%(3)$ & $\mathrm{p}=0.120$ \\
\hline \multicolumn{5}{|l|}{ Premature } \\
\hline CHD Hx & $47.06 \%(8)$ & $100 \%(4)$ & $12.00 \%(3)$ & $\mathrm{p}=0.001$ \\
\hline MAP Use & $0.00 \%$ & $0.00 \%$ & $4.35 \%(1)$ & $\mathrm{p}=1.00$ \\
\hline
\end{tabular}

(Fisher's exact T-test, 95\% C.I) $\alpha .05$

The primary composite clinical endpoint of MI, unstable angina, CHF, need for revascularization and all-cause mortality was found to be also significantly higher in patients with high hs-CRP levels (hs-CRP> $10 \mathrm{mg} / \mathrm{L}$ ). (Table 4)

Table 4. Hs-CRP levels and 30-day clinical outcomes

\begin{tabular}{llll}
\hline CRP Level & $\begin{array}{l}<\mathbf{1 0} \mathbf{~ m g} / \mathbf{L} \\
(\mathbf{n}=\mathbf{2 3})\end{array}$ & $\begin{array}{l}>\mathbf{1 0} \mathbf{~ m g} / \mathbf{L} \\
(\mathbf{n}=\mathbf{1 4})\end{array}$ & \\
\hline Primary Composite Endpoint & $34.78 \%(8)$ & $85.71 \%(12)$ & $\mathrm{p}=0.045$ \\
MI & $0.00 \%$ & $7.14 \%(1)$ & $\mathrm{p}=0.378$ \\
UA & $0.00 \%$ & $14.29 \%(2)$ & $\mathrm{p}=0.137$ \\
CHF & $8.70 \%(2)$ & $21.43 \%(3)$ & $\mathrm{p}=0.346$ \\
Revascularization & $13.04 \%(3)$ & $14.29 \%(2)$ & $\mathrm{p}=1.00$ \\
All-Cause Mortality & $13.0 \%(3)$ & $28.57 \%(4)$ & $\mathrm{p}=0.390$ \\
\hline (Fisher's exact T-test, 95\% C.I) $\alpha .05$ & &
\end{tabular}

Analyzing clinical outcomes using lower CRP cutoffs as recommended by $\mathrm{CDC}$, showed no association between clinical outcomes and CRP in our population. (Table 5)

Table 5. Hs-CRP levels (CDC cutoffs) and 30-day clinical outcomes

\begin{tabular}{llrrl}
\hline CRP Level & $\begin{array}{l}<\mathbf{1 ~ m g} / \mathbf{L} \\
(\mathbf{n}=\mathbf{1 7})\end{array}$ & $\begin{array}{l}\mathbf{1 - 3} \mathbf{~ m g} / \mathbf{L} \\
(\mathbf{n}=5)\end{array}$ & $\begin{array}{l}>\mathbf{3} \mathbf{~ m g} / \mathbf{L} \\
(\mathbf{n}=\mathbf{2 7})\end{array}$ & \\
\hline $\begin{array}{l}\text { Primary Composite } \\
\quad \text { Endpoint }\end{array}$ & $53 \%(9)$ & $20 \%$ & $48 \%$ & $\mathrm{p}=0.497$ \\
MI & $0 \%$ & $0 \%$ & $4 \%$ & $\mathrm{p}=0.660$ \\
UA & $0 \%$ & $0 \%$ & $7 \%$ & $\mathrm{p}=0.610$ \\
CHF & $6 \%(17)$ & $20 \%$ & $11 \%$ & $\mathrm{p}=0.635$ \\
Revasc & $24 \%(4)$ & $0 \%$ & $15 \%$ & $\mathrm{p}=0.479$ \\
All-Cause Mortality & $24 \%(4)$ & $0 \%$ & $11 \%$ & $\mathrm{p}=0.326$ \\
\hline
\end{tabular}

(Fisher's exact T-test, 95\% C.I) $\alpha .05$

\section{Discussion}

High sensitivity C-reactive protein (hs-CRP) levels measured with a high-sensitivity assay is $<1.0 \mathrm{mg} / \mathrm{dl}$ in $98 \%$ of healthy persons. Even when relatively elevated within this "normal" range, CRP has been shown to be predictive of a first cardiovascular event in previously healthy men and women. ${ }^{1,2}$

High sensitivity C-reactive protein (hs-CRP) is one of the chemical markers that has earned much attention in profiling patients with coronary disease. As some literature has spawned its use in prognosticating chronic stable angina, recent studies cite its use in profiling the future clinical courses of patients with acute myocardial infarction. In the TACTICS-TIMI 18 studies concerning NSTEMI/ Unstable Angina, women are more likely to have elevated hs-CRP; men on the other hand were more likely to have elevated creatinine kinase-MB and troponins. Such genderrelated differences need further studies for verification in the setting of acute myocardial infarction.

High sensitivity-CRP also has been reported to be predictive of future events in patients with established CAD. Across the acute coronary syndromes, hs-CRP levels are associated with increased risk of recurrent events, independent of the presence or absence of myocyte necrosis. Hs-CRP levels within 6 hours after the onset of AMI reflect the vulnerability of culprit coronary lesions. A recent study demonstrated that there appears to have positive correlation between hs-CRP levels and increased number of thin cap atheromas in the coronary tree that are vulnerable to rupture. ${ }^{3}$ This might be due to the fact that CRP not only reflects an underlying inflammatory process in the atherosclerotic plaque lesion ${ }^{4}$ but also directly participates in the promotion of atherosclerotic processes and endothelial cell inflammation. ${ }^{5}$

After an acute myocardial infarction, hs-CRP dramatically increases starting approximately 6 hours after the onset of symptoms, peaking in 2 to 4 days and returning to baseline in 3 to 4 weeks. $^{6}$ Peak hs-CRP levels are associated with the size of the infarct and are attenuated by early reperfusion. ${ }^{7}$ Peak hs-CRP levels have been reported to predict early post-AMI events, but prognostic utility from later events has been uncertain. Pietila et al. ${ }^{8}$ evaluated CRP daily after AMI. Peak hs-CRP predicted mortality for up to 6 months, but was not predictive for months 6 through 24 . Anzai et al. ${ }^{9}$ reported that peak hs-CRP levels predicted risk of cardiac death for up to 1 year. Tommasi et al. ${ }^{10}$ reported that hs-CRP, measured 8 hours after AMI, predicted cardiac events at 1 year in 64 patients with normal ejection fraction.

In our study, a high hs-CRP level (>10 mg/L) was found to be positively correlated with an increase in cardiac events, specifically our primary composite endpoint of MI, unstable angina, revascularization, congestive heart failure and allcause mortality. This supports previous data found in medical literature that high levels of hs-CRP can be used for 
the risk stratification of Acute Coronary Syndrome (ACS) patients. Higher levels of hs-CRP immediately post ACS, may be a useful prognosticator of poorer cardiovascular outcomes.

\section{Study Limitations}

Initial sample size computed for the study to be powered adequately was 444 patients. Being an inflammatory marker, there are a variety of confounding factors which would affect hs-CRP levels in the serum. In our attempt to provide a clear and definite picture of the role of hs-CRP in post MI patients, we designed a strict set of exclusion criteria to eliminate such confounders. Unfortunately, we found it very difficult to reach the target sample size due to the stringent exclusion criteria in the protocol.

\section{Recommendations}

1. Explore the association of smoking and increased hsCRP levels in a larger population.

2. An adequately powered study to be able to do univariate and multivariate analyses to test the association of smoking and familial history of premature CHD with hs-CRP levels.

3. Hs-CRP remains a promising marker for higher cardiovascular risk stratification in ACS and warrants further investigation in larger studies.

\section{References}

1. Ridker PM, Hennekens CH, Buring JE, Rifai N. C-reactive protein and other markers of inflammation in the prediction of cardiovascular disease in women. N Engl J Med. 2000; 342(12):836-43.

2. Ridker PM, Cushman M, Stampfer MJ, Tracy RP, Hennekens CH. Inflammation, aspirin and the risk of cardiovascular disease in apparently healthy men. N Engl J Med. 1997; 336(14):973-9.

3. Tomoda H, Aoki N. Prognostic value of C-reactive protein levels within six hours after the onset of acute myocardial infarction. Am Heart J. 2000; 140(2):324-8

4. $\quad$ Ross R. Atherosclerosis - an inflammatory disease. N Engl J Med. 1999; 340(2):115-26

5. Pasceri V, Willerson JT, Yeh ET. Direct proinflammatory effect of Creactive protein on human endothelial cells. Circulation, 2000; 102(18):2165-8.

6. Kushner I, Broder ML, Karp D. Control of the acute phase response. Serum C-reactive protein kinetics after acute myocardial infarction. J Clin Invest. 1978; 61(2):235-42.

7. Pietila K, Hermens WT, Harmoinen A, et al. Comparison of peak serum C-reactive protein and hydroxybutyrate dehydrogenase levels in patients with acute myocardial infarction treated with alteplase and streptokinase. Am J Cardiol. 1997; 80(8):1075-7.

8. Pietila KO, Harmoinen AP, Jokiniitty J, Pasternack AI. Serum C-reactive protein concentration in acute myocardial infarction and its relationship to mortality during 24 months of follow-up in patients under thrombolytic treatment. Eur Heart J. 1996; 17(9):1345-9.

9. Anzai T, Yoshikawa T, Shiraki H, et al. C-reactive protein as a predictor of infarct expansion and cardiac rupture after a first Q-wave acute myocardial infarction. Circulation. 1997; 96(3):778-84.

10. Tommasi S, Carluccio E, Bentivoglio M, et al. C-reactive protein as a marker for cardiac ischemic events in the year after a first, uncomplicated myocardial infarction. Am J Cardiol. 1999; 83(12):1595-9.

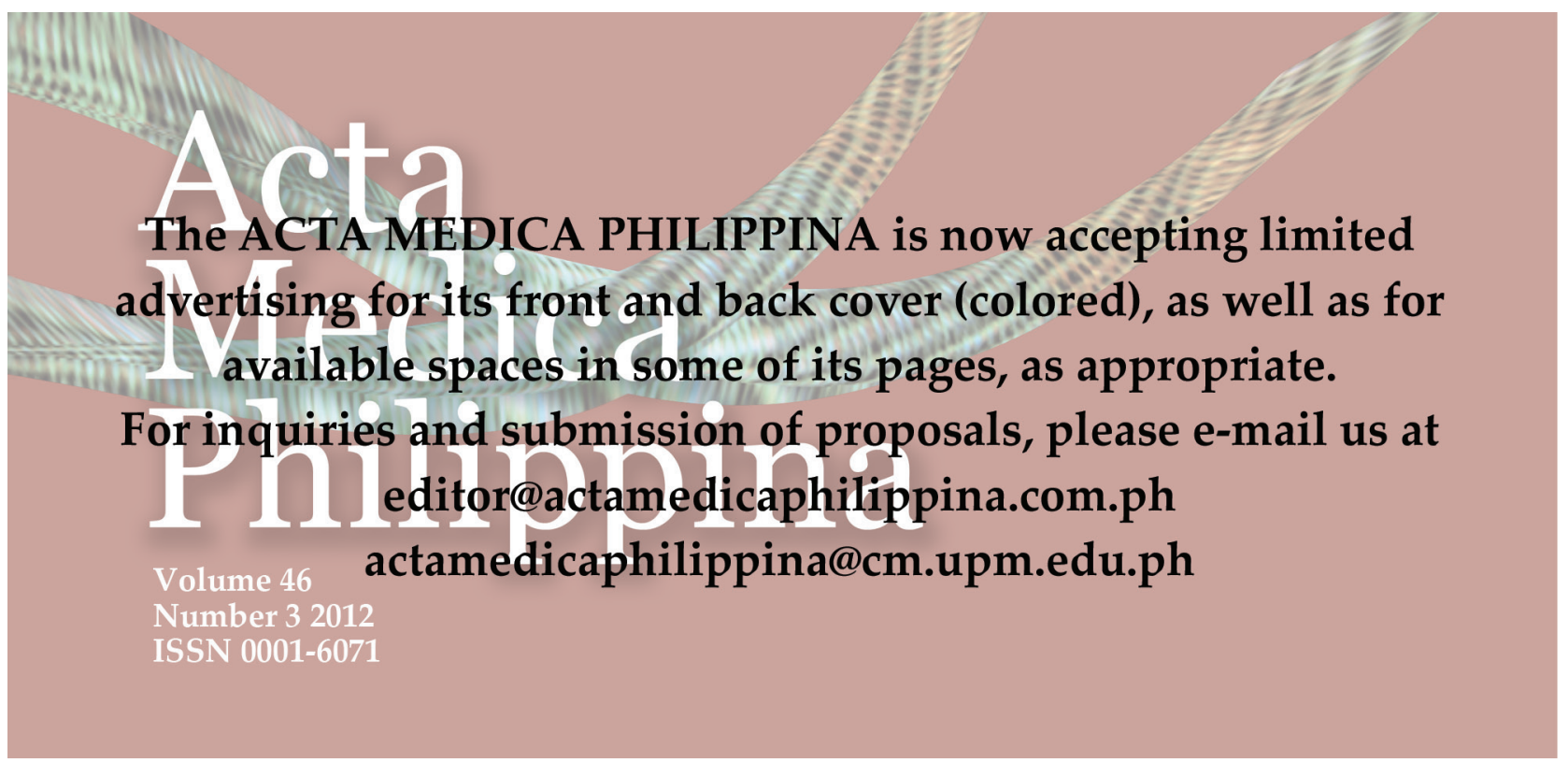

